\title{
Capacidade de reidratação de Lilium pumilum Redouté ${ }^{(1)}$
}

\author{
MIRELLE NAYANA de S. SANTOS ${ }^{(2)^{*}}$; MÁRCIA MARTINS TOLENTINO(2); \\ RITA de CÁSSIA LIMA DE OLIVEIRA ${ }^{(2)}$ e ANA MARIA MAPELI(2)
}

\begin{abstract}
RESUMO
Dentre as flores ornamentais comercializadas como flor de vaso ou corte destaca-se Lilium pumilum, pertencente à família Liliaceae, a qual se caracteriza por apresentar hastes contendo inflorescências com diversos botões florais, fácil reprodução e coloração variada. A comercialização de flores de corte é uma atividade promissora, entretanto o transporte exige atenção, devido ao estresse hídrico que pode prejudicar a absorção de água e posterior reidratação, originando um balanço hídrico negativo, visto que a taxa de absorção é menor que a taxa de transpiração. Sendo assim, a utilização de técnicas adequadas que possibilitem prolongar a longevidade das flores é necessária. Este trabalho teve como objetivo analisar o efeito do tempo de armazenamento a seco na recuperação da turgescência de L. pumilum. Para a condução do experimento, hastes florais de L. pumilum foram armazenadas a seco por $0,12,24,36$ e 48 horas. Ao final de cada período, as inflorescências retornaram à água, sendo analisadas, a cada 6 horas, quanto às alterações no teor relativo de água das tépalas (flor), botão alaranjado e folhas. Diariamente, avaliou-se perda de massa fresca e longevidade. No teor relativo de água da flor observou-se uma queda acentuada durante os períodos de armazenamento a seco, mas com recuperação após seis horas em água, com maior eficiência no tratamento de 48 horas, com recuperação de 86,8\% em relação ao controle. Quanto ao teor relativo de água do botão, em todos os tratamentos houve recuperação. Em relação à variação de massa fresca, observou-se alteração de acordo com o período de armazenamento a seco, sendo que apenas as hastes que permaneceram por 12 e 24 horas a seco obtiveram recuperação superior a sua perda de massa fresca inicial, nas primeiras seis horas de hidratação, em 118,7 e 26,01\%, respectivamente. A longevidade média das hastes não foi alterada (6,8 dias) por nenhum tratamento. Portanto, independente da duração do armazenamento a seco, houve recuperação da turgescência, indicando que hastes de L. pumilum podem ser transportadas a seco por períodos de até 48 horas.
\end{abstract}

Palavras-chave: lírio, estresse hídrico, teor relativo de água, longevidade.

\section{Capacity of rehydration of Lilium pumilum Redouté}

Among the ornamental flowers marketed as flower vase or cut, there is Lilium pumilum belonging to the Liliaceae family, which is characterized by having several stems with inflorescences containing buds, easy propagation and varied coloring. The marketing of cut flowers is a promising activity; however the transport demands attention due to water stress, which can impair the absorption of water and rehydration, yielding a negative water balance, since the absorption rate is less than the rate transpiration. Based on this assumption, the use of appropriate techniques that allow extending the longevity of the flowers is required. This study aimed to analyze the effect of storage time on the recovery of dry turgor L. pumilum. For this flower stems were dry-stored for $0,12,24,36$ and 48 hours. At the end of each period, the inflorescences returned to the water, and analyzed every 6 hours for changes in relative water content of the tepals (flower), orange buds and leaves. Daily evaluations were changes of weight and longevity. The relative water content of flower there was a sharp drop during periods of dry storage, but with recovery after 6 hours in water with greater efficiency in the treatment of 48 hours, with recovery of $86.8 \%$ relative to the control. As the relative water content of the button, all treatments were recovered. Regarding the variation of fresh mass, there was variation with the period of dry storage, and only the stems that remained for 12 to 24 hours to dry obtained superior recovery for loss of initial fresh weight within the first six hours of hydration at 118.7 and $26.01 \%$, respectively. The average longevity of the stems was unchanged (6.8 days) for no treatment. Therefore, regardless of the duration of dry storage, there was recovery of turgor, indicating that flower stems of L. pumilum can be transported dry for periods up to 48 hours.

Keywords: Lily, water stress, relative water content, longevity.

\section{INTRODUÇÃO}

Atualmente, a floricultura é considerada uma atividade econômica de grande relevância no agronegócio, com indicadores de crescimento positivos em termos de número de produtores, área cultivada e Valor Bruto da Produção (VBP), sendo que a cadeia produtiva de flores e plantas ornamentais no Brasil movimentou no ano de 2013, o valor global de R $\$ 6$ bilhões, acumulando crescimento de 8,3\% sobre os resultados obtidos no ano de 2012 (JUNQUEIRA e PEETZ, 2014). Contudo, a floricultura enfrenta o problema da alta perecibilidade das flores, havendo a necessidade do desenvolvimento e uso de técnicas que promovam a durabilidade das mesmas, mantendo a qualidade do

\footnotetext{
(1) Recebido em 08/09/2015 e aceito em 03/02/2016

(2) Universidade Federal do Oeste da Bahia, Centro das Ciências Biológicas e da Saúde, Barreiras-BA, Brasil. * Autor correspondente: mirellebio@ hotmail.com
} 
produto e reduzindo as perdas pós-colheita (SONEGO e BRACKMANN, 1995).

Dentre as flores ornamentais comercializadas, as espécies do gênero Lilium L., pertencente à família Liliaceae, possuem ampla distribuição em ambientes temperados, tendo como principais características a presença de bulbos e raízes rizomatosas, folhas alternas e espiraladas, além de haste contendo inflorescência com variados botões florais, cuja coloração é diversificada, podendo ser laranja, amarelo, branco, vermelho ou rosa (JUDD et al., 2009). Dentro deste gênero inclui-se Lilium pumilum Redouté, que destaca-se no agronegócio em virtude do seu valor ornamental, além de se reproduzir facilmente e proporcionar o cultivo de variedades com flores perfumadas, de forma e colorido variados, com florescimento vistoso, sendo as flores grandes, alaranjadas, terminais, em grupos de 2-5, podendo ser comercializada como flor de corte ou de vaso (JUDD et al., 2009; LORENZI e SOUZA, 2008).

Vale destacar que a longevidade das flores de corte está associada aos fatores fisiológicos inerentes da espécie, como a ação do hormônio etileno, e aos fatores ambientais, como temperatura, umidade e qualidade da água do vaso. $\mathrm{O}$ desequilíbrio entre a absorção de água pela haste e transpiração, causado pela obstrução física dos vasos xilemáticos, reduz a disponibilidade de água, ocorrendo murcha das pétalas e estímulo da produção de etileno (DIAS-TAGLICOZZO et al., 2005), sendo um dos principais problemas observados na comercialização de flores de corte.

A perda excessiva de água está associada ao período que as hastes florais permanecem a seco (SPRICIGO et al., 2012), na maioria das vezes devido ao longo trajeto até o local de beneficiamento. Partindo disso, a reidratação das hastes florais visa restaurar os danos ocorridos nas flores de corte durante o transporte e o armazenamento a seco, restabelecendo a sua turgidez (SUZUKI et al., 2001; STRINGUETA et al., 2002) e prolongando a longevidade das mesmas.

Assim, este trabalho teve como objetivo analisar o efeito do tempo de armazenamento a seco na recuperação da turgescência de L. pumilum.

\section{MATERIAL E MÉTODOS}

As hastes florais de L. pumilum foram fornecidas por um floricultor de Brasília-DF, devido à inexistência de produtores na região oeste da Bahia. As hastes florais de lírio foram adquiridas envasadas, permanecendo 12 horas em câmara fria $\left(5^{\circ} \mathrm{C}\right)$, para aclimatação. Os experimentos ocorreram no Laboratório de Botânica, do Instituto de Ciências Ambientais e Desenvolvimento Sustentável (ICADS), da Universidade Federal da Bahia, durante o mês de abril de 2012 a janeiro de 2013.

As hastes de lírio foram cortadas em laboratório e padronizadas quanto ao comprimento $(25 \mathrm{~cm})$, ausência de danos mecânicos e ataque de patógenos. Em seguida, determinou-se a capacidade de reidratação das hastes florais de L. pumilum após armazenamento a seco por 12, 24, 36 e 48 horas, sendo que as hastes controle permaneceram sempre em água destilada. Ao final de cada período, as inflorescências retornaram à água, onde permaneceram até o final do experimento, sob temperatura de $25^{\circ} \mathrm{C}$, umidade relativa de $50-70 \%$ e intensidade luminosa de 7-10 $\mu \mathrm{mol}$ $\mathrm{m}^{-2} \mathrm{~s}^{-1}$, com duração variável de acordo com o aspecto visual aceitável para o consumidor.

Diariamente, avaliou-se: - longevidade das flores: número de dias compreendidos entre o corte e o momento da perda da qualidade da flor aberta (BARBOSA et al., 2006); - perda de massa fresca: as hastes foram pesadas ao final de cada período de armazenamento a seco $(0,12,24$, 36 e 48 horas) e após 6, 12, 18 e 24 horas de reidratação. Antes de serem submetidas ao armazenamento a seco, foi atribuída uma perda de massa inicial de $0 \%$. Os valores foram adquiridos de acordo com a equação: $\mathrm{PMF}=(\mathrm{Mf}$ x 100) / Mi -100, em que: PMF: Perda de massa fresca em \%; Mf: Massa fresca final das hastes (g); Mi: Massa fresca inicial das hastes $(\mathrm{g})$; - teor relativo de água (TRA): determinado ao final do período de armazenamento a seco e 6,12,18 e 24 horas após as hastes retornarem à água. Em função do tamanho das tépalas, foram retirados discos $(0,5 \mathrm{~cm})$ das mesmas e agrupados em cinco, o mesmo ocorreu com botão e folha. Os mesmos foram pesados, para obtenção da massa fresca e colocados em espuma de poliuretano saturada com água, onde permaneceram por seis horas, sendo retirados e pesados para determinação da massa túrgida. Em seguida, os discos foram mantidos em estufa a $65^{\circ} \mathrm{C}$, por 24 horas, para obtenção da massa seca constante. O TRA foi medido de acordo com a equação proposta por Catsky (1974): TRA $=((\mathrm{F}-\mathrm{W}) /(\mathrm{T}-\mathrm{W})) \mathrm{x}$ 100 , em que: $\mathrm{F}=$ massa fresca $(\mathrm{g}) ; \mathrm{W}=$ massa seca $(\mathrm{g}) ; \mathrm{T}$ = massa túrgida $(\mathrm{g})$.

O experimento foi conduzido em delineamento experimental em blocos casualizados, com 5 repetições, tendo-se 2 hastes por unidade experimental. Os dados foram interpretados usando análise de variância e as médias comparadas pelo Teste de Scott-Knott ao nível de 5\% de probabilidade.

\section{RESULTADOS E DISCUSSÃO}

A partir das análises, verificou-se que independente do tempo de armazenamento a seco, nenhum tratamento alterou a longevidade, a qual teve uma média de 6,8 dias (Figura 1). Os resultados obtidos divergem do que foi verificado em Epidendrum ibaguense, pois o armazenamento a seco, por 36 e 48 horas, proporcionou redução significativa na vida de vaso das inflorescências, sendo que no tratamento de 48 horas não houve recuperação das hastes após reidratação (SANTOS et al., 2012). Assim, as espécies diferem quanto à capacidade de resistência ao armazenamento a seco, o que pode influenciar na reidratação e, consequentemente, na durabilidade das hastes florais. 


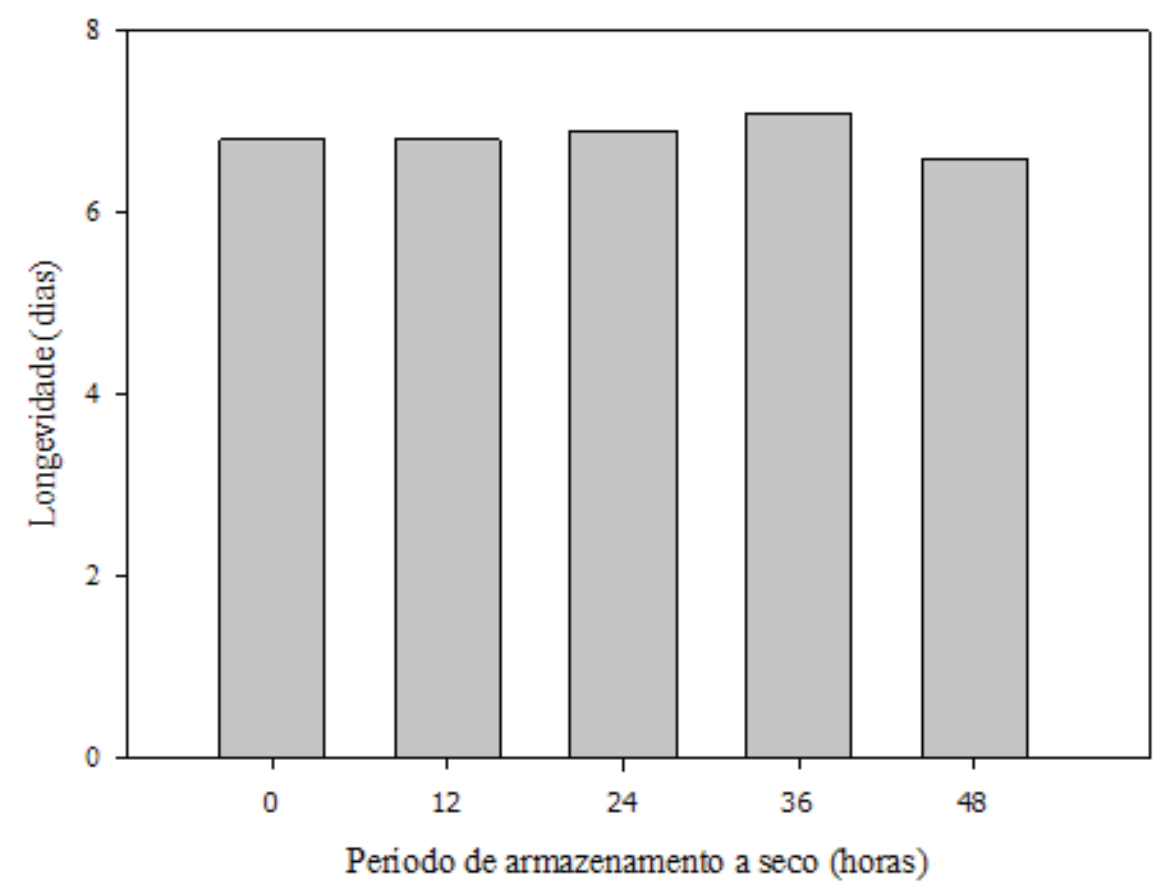

Figura 1. Longevidade de inflorescências de Lilium pumilum submetidas ao armazenamento a seco.

Figure 1. Longevity inflorescences of Lilium pumilum submitted to dry storage.

Em relação à perda de massa fresca, observou-se que o efeito foi dependente do período de armazenamento a seco, sendo as maiores perdas nos períodos mais longos de déficit hídrico (Figura 2), provavelmente ocasionadas pelo balanço hídrico desfavorável, uma vez que a absorção foi interrompida e a transpiração mantida.

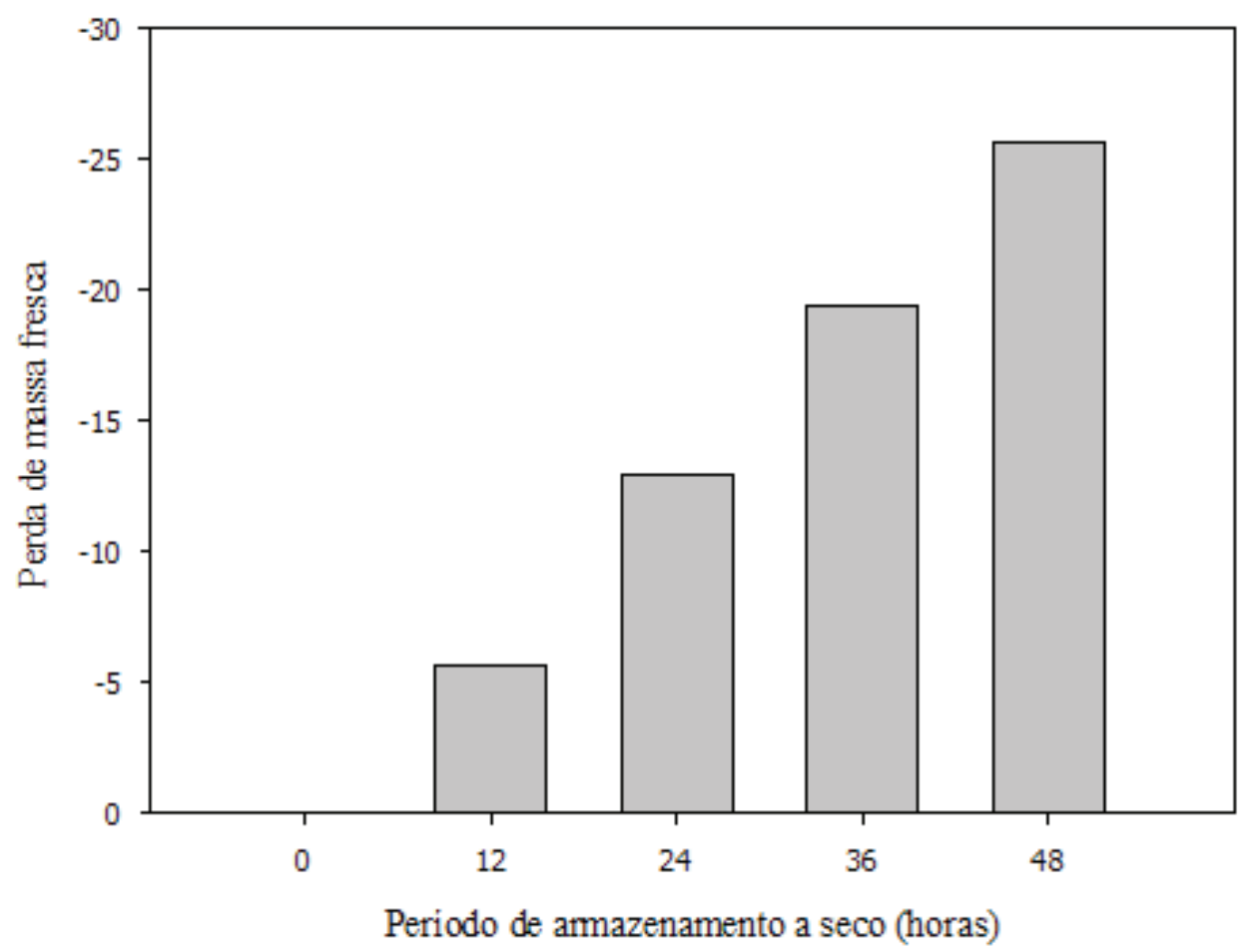

Figura 2. Perda de massa fresca de inflorescências de Lilium pumilum submetidas ao armazenamento a seco.

Figure 2. Loss of fresh mass of inflorescences of Lilium pumilum submitted to dry storage. 
Logo após serem colocadas em água, as hastes florais apresentaram incrementos em sua matéria fresca, indicando ampla taxa de absorção de água nas seis primeiras horas de hidratação, mantendo-se até 12 horas, independente do tempo de armazenamento a seco (Figura
3). No entanto, somente as hastes que permaneceram por 12 e 24 horas a seco obtiveram recuperação superior a sua perda de massa fresca inicial (Figura 3), nas primeiras seis horas de hidratação, em 118,7 e 26,01\%, respectivamente.

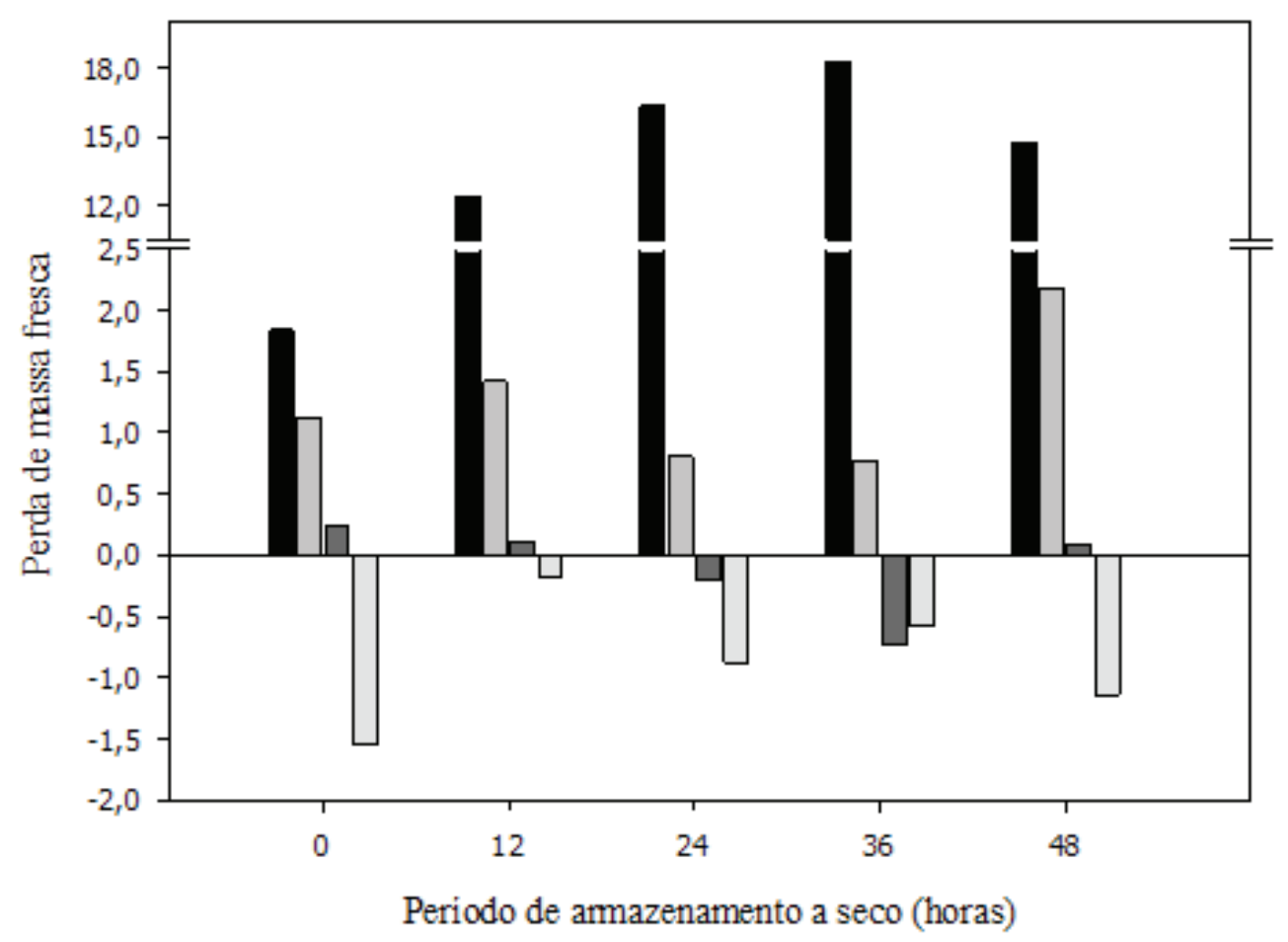

Figura 3. Perdas de massa fresca de inflorescências de Lilium pumilum submetidas ao armazenamento a seco por 12, 24, 36 e 48 horas. O tempo de reidratação é simbolizado por $(<)$ 6 horas, $(<) 12$ horas, $(<) 18$ horas e $(>) 24$ horas.

Figure 3. Loss of fresh mass of inflorescences of Lilium pumilum submitted to dry storage for 12, 24, 36 and 48 hours. The rehydration time is represented by $(<) 6$ hours, $(<) 12$ hours, $(<) 18$ hours and (<) 24 hours.

Já nas hastes com 36 e 48 horas de armazenamento a seco, a reidratação foi incompleta, com recuperação de $-5,9$ e $-49,3 \%$ da massa fresca inicial das inflorescências, respectivamente (Figuras 2 e 3). Provavelmente, hastes armazenadas a seco por períodos maiores apresentam interrupção da coluna de água nos vasos condutores, o que reduz a absorção de água (VAN MEETEREN et al., 2005), sendo que a intensidade de inibição depende da espécie.

Santos et al. (2012), trabalhando com orquídea E. ibaguense, observaram que todas as hastes florais submetidas ao estresse hídrico, nas primeiras 4 horas de reidratação, absorveram água, independente do tempo de armazenamento a seco, mas só houve recuperação completa da massa fresca no tratamento de 12 horas a seco. Comportamento semelhante foi observado para Ave-do-Paraíso (Strelitzia reginae), pois todas as hastes, independente do período de armazenamento a seco, absorveram água durante as seis primeiras horas, no entanto, somente as hastes que permaneceram por 16 horas a seco tiveram recuperação completa da massa fresca inicial (KARSTEN et al., 2013). Já em rosas
(Rosa hybrida L.) cv. Samantha, submetidas 24 horas ao estresse hídrico, houve perda de $28,8 \%$ da massa inicial, a qual não foi recuperada, mesmo após terem sido colocadas em água por 2 horas (JIN et al., 2006). Esse resultado demonstra que esta espécie de rosa pode ser mais sensível ao estresse hídrico do que L. pumilum ou que o tempo de reidratação foi muito pequeno, sendo considerado insuficiente.

Os efeitos observados na redução da massa fresca das inflorescências de L. pumilum foram confirmados ao analisar o teor relativo de água da flor (tépalas), botão e folha, pois houve redução devido ao armazenamento a seco, sendo a queda proporcional ao tempo de estresse hídrico na flor e no botão (Tabelas 1, 2 e 3). A taxa de absorção variou em todos os tratamentos, mas com aumento após seis horas em reidratação, como constatado na variação de massa fresca.

No teor relativo da água das tépalas, observou-se a queda acentuada durante os períodos de armazenamento a seco, mas com recuperação após as seis horas em água (Tabela 1). Os tratamentos com 12 e 24 horas de estresse, 
promoveram recuperação comparado ao seu valor inicial de 9,14 e $56,1 \%$, em relação às seis horas de reidratação. Nas hastes armazenadas a seco por 36 horas, o incremento significativo na hidratação das tépalas ocorreu após 18 horas de armazenamento úmido. $\mathrm{O}$ tratamento com 48 horas de estresse promoveu recuperação de $86,8 \%$ do teor relativo de água nas primeiras seis horas de reidratação, em relação ao seu valor inicial, sendo que o controle se manteve estável durante as 24 horas de hidratação. Observou-se, então, que flores de L. pumilum conseguiram recuperar boa parte de sua turgescência, mesmo quando armazenadas a seco por 48 horas (Tabela 1).

Tabela 1. Teor Relativo de Água (\%) de tépalas de Lilium pumilum submetidas aos diferentes períodos de armazenamento a seco $(0,12,24,36$ e 48 horas), em função do tempo de reidratação (0, 6, 12, 18, 24 horas).

Table 1. Relative Water Content (\%) of Lilium pumilum petals submitted to different periods of dry storage (0, 12, 24, 36 and 48 hours), depending on the rehydration time (0, 6, 12, 18, 24 hours).

\begin{tabular}{|c|c|c|c|c|c|}
\hline \multirow[t]{2}{*}{ Período de Armazenamento (horas) } & \multicolumn{5}{|c|}{ Tempo de reidratação (horas) } \\
\hline & 0 & 6 & 12 & 18 & 24 \\
\hline Controle & $78,25 \mathrm{~A}$ & $77,30 \mathrm{~A}$ & $92,20 \mathrm{~A}$ & $73,45 \mathrm{~A}$ & $91,99 \mathrm{~A}$ \\
\hline 12 & $69,12 \mathrm{~B}$ & $75,44 \mathrm{~A}$ & $81,49 \mathrm{~A}$ & $79,28 \mathrm{~A}$ & $69,32 \mathrm{~B}$ \\
\hline 24 & $53,74 \mathrm{D}$ & $83,88 \mathrm{~A}$ & $60,30 \mathrm{C}$ & $76,53 \mathrm{~B}$ & $64,81 \mathrm{C}$ \\
\hline 36 & $54,41 \mathrm{~B}$ & $64,04 \mathrm{~B}$ & $53,82 \mathrm{~B}$ & $75,04 \mathrm{~A}$ & $77,46 \mathrm{~A}$ \\
\hline 48 & $34,04 \mathrm{C}$ & $63,54 \mathrm{~B}$ & $75,45 \mathrm{~A}$ & $80,85 \mathrm{~A}$ & $83,23 \mathrm{~A}$ \\
\hline
\end{tabular}

Médias seguidas pela mesma letra, na linha, não diferem entre si pelo teste Scott-Knott ao nível de $5 \%$ de probabilidade.

Means followed by the same letter, in the line, do not differ by Scott-Knott test at 5\% probability.

Santos et al. (2012) e Karsten et al. (2013) encontraram resultados diferenciados em E. ibaguense e $S$. reginae, respectivamente, onde a primeira só teve capacidade de recuperação de boa parte da turgescência quando armazenada a seco até 12 horas; na segunda espécie, a recuperação do teor relativo de água só ocorreu até as 16 horas a seco. A não recuperação da turgescência nos demais tratamentos a seco pode estar relacionada ao bloqueio dos vasos xilemáticos. Sugere-se, então, que o bloqueio dos vasos na espécie $L$. pumilum seja baixo até as 48 horas de armazenamento a seco, o que proporciona uma vantagem quanto ao tempo de transporte até o local de beneficiamento, diminuindo a perda da mesma, proporcionando uma maior exportação.

Quanto ao teor relativo de água do botão, determinou-se queda marcante no decorrer do tempo de armazenamento a seco, sendo que houve recuperação nos tratamentos de 24 e 48 horas, após seis horas de hidratação em 53\% e 47\% respectivamente, e 36 horas, após 18 horas de hidratação, não ocorrendo o mesmo com o tratamento de 12 horas a seco, pois não houve recuperação significativa em nenhum período de reidratação, comparado ao seu valor inicial (Tabela 2).

Tabela 2. Teor Relativo de Água (\%) dos botões de Lilium pumilum, submetidos aos diferentes períodos de armazenamento a seco $(0,12,24,36$ e 48 horas) em função do tempo de reidratação $(0,6,12,18,24$ horas).

Table 2. Relative Water Content (\%) of Lilium pumilum buttons submitted to different periods of dry storage (0, 12, 24, 36 and 48 hours), depending on the rehydration time $(0,6,12,18,24$ hours).

\begin{tabular}{|c|c|c|c|c|c|c|}
\hline Período de Armazenamento (horas) & \multicolumn{5}{|c|}{ Tempo de reidratação (horas) } \\
\hline Controle & $82,19 \mathrm{~A}$ & $90,16 \mathrm{~A}$ & $87,46 \mathrm{~A}$ & $84,75 \mathrm{~A}$ & $86,61 \mathrm{~A}$ \\
\hline 12 & $95,28 \mathrm{~A}$ & $87,07 \mathrm{~B}$ & $85,72 \mathrm{~B}$ & $87,08 \mathrm{~B}$ & $74,89 \mathrm{~B}$ \\
\hline 24 & $71,53 \mathrm{~B}$ & $83,05 \mathrm{~A}$ & $71,41 \mathrm{~B}$ & $78,24 \mathrm{~A}$ & $67,50 \mathrm{~B}$ \\
\hline 36 & $69,95 \mathrm{~B}$ & $73,33 \mathrm{~B}$ & $72,21 \mathrm{~B}$ & $78,48 \mathrm{~A}$ & $83,10 \mathrm{~A}$ \\
\hline 48 & $48,59 \mathrm{~B}$ & $68,49 \mathrm{~A}$ & $78,36 \mathrm{~A}$ & $75,68 \mathrm{~A}$ & $74,74 \mathrm{~A}$ \\
\hline
\end{tabular}

Médias seguidas pela mesma letra, na linha, não diferem entre si pelo teste Scott-Knott ao nível de $5 \%$ de probabilidade.

Means followed by the same letter, in the line, do not differ by Scott-Knott test at 5\% probability. 
No teor relativo de água da folha (Tabela 3), houve aumento de hidratação nas seis primeiras horas de reidratação, nos tratamentos de 12, 24, 36 e 48 horas de hidratação. No tratamento com 48 horas de estresse, houve maior incremento de $17,45 \%$ em relação ao seu valor inicial, nas seis primeiras horas de hidratação, comparando com o controle e demais tratamentos $0,12,24$ e 36 horas com 12,$25 ; 15,76 ; 14,18 ; 9,64 \%$ (Tabela 3 ).

Tabela 3. Teor Relativo de Água (\%) da folha de Lilium pumilum submetidas aos diferentes períodos de armazenamento a seco $(0,12,24,36$ e 48 horas), em função do tempo de reidratação $(0,6,12,18,24$ horas).

Table 3. Relative Water Content (\%) of Lilium pumilum leaves submitted to different periods of dry storage (0, 12, 24, 36 and 48 hours), depending on the rehydration time (0, 6, 12, 18, 24 hours).

\begin{tabular}{|c|c|c|c|c|c|}
\hline Período de Armazenamento (horas) & \multicolumn{4}{|c|}{ Tempo de reidratação (horas) } \\
\hline & 0 & 6 & 12 & 18 \\
\hline Controle & $82,19 \mathrm{~A}$ & $90,16 \mathrm{~A}$ & $87,46 \mathrm{~A}$ & $84,75 \mathrm{~A}$ & $86,61 \mathrm{~A}$ \\
\hline 12 & $95,28 \mathrm{~A}$ & $87,07 \mathrm{~B}$ & $85,72 \mathrm{~B}$ & $87,08 \mathrm{~B}$ & $74,89 \mathrm{~B}$ \\
\hline 24 & $71,53 \mathrm{~B}$ & $83,05 \mathrm{~A}$ & $71,41 \mathrm{~B}$ & $78,24 \mathrm{~A}$ & $67,50 \mathrm{~B}$ \\
\hline 36 & $69,95 \mathrm{~B}$ & $73,33 \mathrm{~B}$ & $72,21 \mathrm{~B}$ & $78,48 \mathrm{~A}$ & $83,10 \mathrm{~A}$ \\
\hline 48 & $48,59 \mathrm{~B}$ & $68,49 \mathrm{~A}$ & $78,36 \mathrm{~A}$ & $75,68 \mathrm{~A}$ & $74,74 \mathrm{~A}$ \\
\hline
\end{tabular}

Médias seguidas pela mesma letra, na linha, não diferem entre si pelo teste Scott-Knott ao nível de 5\% de probabilidade.

Means followed by the same letter, in the line, do not differ by Scott-Knott test at 5\% probability.

A longevidade de L. pumilum não foi afetada pelo armazenamento a seco devido à alta capacidade de reidratação da espécie, tanto no que se refere ao botão e flor, quanto à folha, a qual não apresentou perdas do seu conteúdo hídrico durante o período de estresse.

\section{CONCLUSÃO}

Inflorescências cortadas de L. pumilum podem ser armazenadas a seco por, no mínimo, 48 horas, pois são capazes de recuperar a turgescência, não perdendo seu valor comercial.

\section{REFERÊNCIAS}

BARBOSA, J.G.; MEDEIROS, A.R.S.; FINGER, F.L.; REIS, F.P.; ÁLVARES, V.S.; BARBOSA, M.S. Longevidade de inflorescências de lírio, de diferentes estádios de colheita, pré-tratadas com sacarose e tiossulfato de prata (STS). Ciência Rural, v.36, p.99-104, 2006.

BARBOSA, J.G.; MEDEIROS, A.R.S; FINGER, L.F.; REIS, F.P.; ÁLVARES, V.S.; BARBOSA, M.S. Longevidade de inflorescências de lírio, de diferentes estádios de colheita, pré-tratadas com sacarose e tiossulfato de prata (STS). Ciência Rural, v.36, p.99-104, 2006.

CATSKY, J.W. In: SLAVIK, B. Methods of studying plant water relations. Berlim: Springer - Verlag, p.121$131,1974$.

DIAS-TAGLIACOZZO, G.M.; FINGER, F.L.; BARBOSA, J.G. Fisiologia pós-colheita de flores de corte.
Revista Brasileira de Horticultura Ornamental, v.11, p.89-99, 2005.

JIN, J.; SHAN, N.; MA, N.; BAI, J.; GAO, J. Regulation of ascorbate peroxidase at the transcript level is involved in tolerance to postharvest water deficit stress in the cut rose (Rosa hybrida L.) cv. Samantha. Postharvest Biology and Technology, v.40, p.236-243, 2006.

JUDD, W.S.; CAMPBELL, C.S.; KELLOGG, E.A.; STEVENS, P.F.; DONOGHU, M.J. Sistemática Vegetal: um enfoque filogenético. $3^{\mathrm{a}}$ ed. Trad. André Olmos Simões et al. Porto Alegre: cap.9; 2009.

JUNQUEIRA, A.H.; PEETZ, M.S. O setor produtivo de flores e plantas ornamentais do Brasil, no período de 2008 a 2013: atualizações, balanços e perspectivas. Revista Brasileira de Horticultura Ornamental, v.20, p.115-120, 2014.

KARSTEN, J.; CHAVES, D.V.; FINGER, L.F.; BARBOSA, J.G. Papel e caracterização da polifenoloxidase em hastes de ave-do-paraíso (Strelitzia reginae Ait.). Revista Brasileira de Horticultura Ornamental, v.19, p.161-171, 2013.

LORENZI, H.; SOUZA, H. M. Plantas Ornamentais no Brasil: arbustivas, herbáceas e trepadeiras. 4. Ed. Nova Odessa, SP: Instituto Plantarum, p.738-739, 2008.

SANTOS, J.S.; FINGER, F.L.; VIEIRA, L.M.; MENDES, T.D.C.; BARBOSA, J.G. Capacidade de reidratação e influência do corte no restabelecimento do fluxo de água em inflorescências de Epidendrum ibaguense. Revista Brasileira de Horticultura Ornamental, v.18, p.15-21, 2012. 
SONEGO, G.; BRACKMANN, A. Conservação póscolheita de flores. Ciência Rural, v.25, p.473-479, 1995.

SPRICIGO, P.C.; FERREIRA, M.D.; CALBO, A.G. Turgescência de crisântemos após a colheita, utilizando o equipamento Wiltemeter. Ciência Rural, v.42, p.225-260, 2012.

STRINGUETA, A.C.O.; LÍRIO, V.S.; SILVA, C.A.B.; REIS, B.S.; AGUIAR, D.R.D. Diagnóstico do segmento de produção da cadeia produtiva de flores plantas ornamentais do Rio de Janeiro. Revista Brasileira de Horticultura Ornamental, v.8, p.77-90, 2002.
SUZUKI, A.; LEONARD, R.T.; NELL, T.A.; BARRETT, J.E.; CLARK, D.G. Effects of retail hydration on water uptake and quality of 'Madame Delbard' roses after long term transport. Acta Horticulturae, v.543, p.251-256, 2001.

VAN MEETEREN U.; VAN GELDER, A.; VAN IEPEREN, W. Effect of growth conditions on post harvest dehydration ability of cut Chrysanthemum Flowers. Acta Horticuture, v.669, p.287-296, 2005. 